\title{
Creation and Usability Evaluating of E-Learning Contents for Automobile Repair Block Painting
}

\author{
Shigeru Ikemoto \\ Graduate School of Science and Technology \\ Kyoto Institute of Technology \\ Kyoto, Japan \\ Yuka Takai \\ Faculty of Design Technology \\ Osaka Sangyo University \\ Daito, Osaka, Japan
}

\author{
Hiroyuki Hamada \\ Future-Applied Conventional Technology Center \\ Kyoto Institute of Technology \\ Kyoto, Japan \\ Noriaki Kuwahara \\ Graduate School of Science and Technology \\ Kyoto Institute of Technology \\ Kyoto, Japan
}

\begin{abstract}
Due to the fact that paintwork in the automobile repair industry requires individual correspondence, work by human hands is indispensable. Although the skills of expert engineers have a great influence on the finish, learning these skills require a lot of experience and time. In Japan, the number of young people in the automobile mechanic and automobile repair industry is drastically decreasing due to the declining birthrates, the trend of young people turning away from driving cars, and the diversification of occupation options. Moreover, the aging of the mechanics and repair technicians has been progressing, and the average age of the mechanics and repair technicians remaining in this industry has been increasing every year. In the near future, there is a high possibility that the shortage of human resources supporting this industry will become apparent. In this study, we aimed to construct a selfstudy support system for young engineers engaged in automobile repair painting to support skill acquisition, using e-learning teaching materials utilizing motion analysis data on block painting by solid paint done by experienced engineers. Furthermore, the usability of the teaching materials was clarified from the viewers' characteristics obtained by publishing the teaching materials. The e-learning teaching materials which secured a certain number of repeaters had a possibility to be effective teaching material. At the same time, several tasks such as the shortening of playback time of the teaching material time were also highlighted.
\end{abstract}

Keywords-Automobile repair; block painting; expert; elearning; usability

\section{INTRODUCTION}

The success of transferring skills is a social problem often spoken in various fields. The work which was once done by human beings has been mechanized and automated by the evolution of various research and technology, but still, there is a lot of work that cannot be done without human intervention. In Japan, problems of successful skill inheritance in the manufacturing industry are particularly noticeable, and the training of young engineers has been a big challenge. Many

This paper was supported by was supported by JSPS KAKENHI Grant (no. 16K16329). efforts and research has been done for successful skill inheritance.

Automobile repair paint work is no exception, there are many tasks done by hand. In the first place, types of damage spots on the cars are unique, they differ case by case, and thus flexible repair techniques based on engineers' experience and reliable technologies are required.

As a general well-known teaching method for capacity building, there is on-job training (OJT) that hones his/her skills while engaging in on-site work. Through specific works, the purpose of OJT is for expert engineers to intentionally and systematically teach the essential knowledge, skills, and attitudes required for the job on a constant basis for inexperienced engineers or beginners. By doing so, it refers to all activities that foster the processing capacity and competence of the overall manufacturing activities [1].

There are also off-JT learning from the field outside the company and schools, but it requires extra funds and time [2]. Considering the nature of the automobile repair and paint industry, which is dominated by small and medium-sized factories hiring less than 10 employees [3] that suffer from financially harsh environments, it is difficult to encourage them to take the Off-JT program. This basically, leaves OJT as the more realistic and reliable method to improve the skill development of their workers.

However, there is a disadvantage to the OJT program. It takes a lot of time to transfer these particular skills, especially related to the workers' long-years of experience and talents. Both skilled and young engineers must carry out OJT while conducting normal operations. For this reason, it is difficult to tackle OJT only at the site. Without the understanding of the management team, including immediate managers or direct bosses in the workplace, it will be difficult to carry out OJT efforts in an effective manner. In order to conduct effective OJT, it is necessary for all employees, including management and others, to recognize that "human resource development (skill succession) is a management task and must be dealt with urgently" [4]. Also, we must consider the disadvantage, [5] that 
the contents and quality of guidance will vary depending on the teaching ability of the people who are acquiring the skills. Being skilled engineers don't always mean that they are excellent leaders, and improvement of the teaching ability of the teaching side is also an important subject. Taguchi explains that the approach of traditional skill transfer is divided into 1) methods mainly for recording and extracting technologies and skills, 2) methods instructed by leaders directly to successors, 3) instructor's support and self-learning, 4) System using IT, and 5) consulting [6]. OJT falls under 2) of these. It can be said that it is important to incorporate methods other than 2) in order to complement the drawbacks of OJT and to carry out more effective skill transfer. In the transferring of skills, IT and digitization are advancing, and Song says elearning is one of the effective tools of teaching methods [7]. There are other studies that incorporate e-learning to acquire the certain skills, such as the studies of skill transferring in welding technology done by $\mathrm{Mr}$. Sou [8], in Kyo wall construction [9] by Takai et al. and in training of new nurses by Isetami et al. [10]. Also, in the United States, e-learning of automobile repair is operated [11], but it is not yet existed in Japan.

We have visualized and digitized techniques essential in skilled technicians in painting work for the purpose of transferring skills [12]. In this research, we created e-learning content for young engineers using this experiment result. Furthermore, usability of contents was clarified from viewers' characteristics obtained by content disclosure.

\section{Collecting And Selecting Data For TeAching MATERIALS}

\section{A. Data Collection Method and Analysis Result}

Engineers engaged in automobile repair painting are required to perform a block painting work using solid paint on a door panel of an automobile. The spray gun's movement and worker's motion was measured and analyzed by the threedimensional movement and also, by the gripping force measuring devices. The painting work process is shown in Fig. 1. In addition, the finishing touch was evaluated by measuring the paint film of the painted panel using a film thickness meter. Details of the experimental methods are as reported in the preceding research [12]. The results obtained by experts compared with non-experts were as follows:

- The standing position did not change and the body did not tilt greatly (see Fig. 2).

- There was a trend that both armpits and elbow were closed.

- The distance between the standing position and the gun was close to the painted object.

- The motion range of the gun was small and the speed was fast.

- The spray gun speed decreased toward the end of the panel (see Fig. 3).

- The spray gun nozzle was held tightly.
- The entire door panel was finished with uniform film thickness.

\section{B. Disclosure of Results to Interview Participants and Hearing Survey}

"Measurement posture of engineer", "angle of right elbow", "angle of right side", "distance between spray gun and door panel", "measurement of distance between hip and door panel", "Spray gun travel distance", "Spray gun travel speed", "Thickness of paint film", "Spray gun trajectory", "Power to grasp spray gun", Expert and non-expert work video to the experiment [12] participants at the meeting. After reporting the result, the experts were asked why they were performing actions that resemble the result. Non-experts were also interviewed and asked at which points they felt were difficult to do in painting work and what were the useful outcomes from this experiment.

At the briefing session, not only the experiment participants but also super experts who taught painting to the experts who participated in the experiment were involved. The super experts pointed out "the test results showed that the experts conducted the painting exactly how they taught". "And the experts who have more than 40 years of work experience are doing their own movements to work better." Considering the fact that the super skilled experts had a lot of knowledge, they had a unique way of painting which was very different but better than the regular experts. The super skilled experts felt it was better to exclude their data and include only the expert's movements for better and accurate results as teaching materials. There was an opinion from experts: "Since I already forgot how I was taught, if there areteaching materials, it will be a guide." And opinion of a non-expert was voiced to take further explanation for his/her motion recorded in the video.

\section{Selection of Contents of E-Learning Material}

Based on the analysis result and remarks of the attendees at the report meeting, we selected the contents of the teaching in the e-learning material. The contents of the teaching in the elearning material were decided as follows:

1) Show a video recorded of an expert painting to trainees.

2) Make sure the posture during painting and a trajectory of the spray gun from the back and side angle.

3) Panel and body position relationship.

4) Operating speed of spray gun.

5) Angle of armpit and elbow.

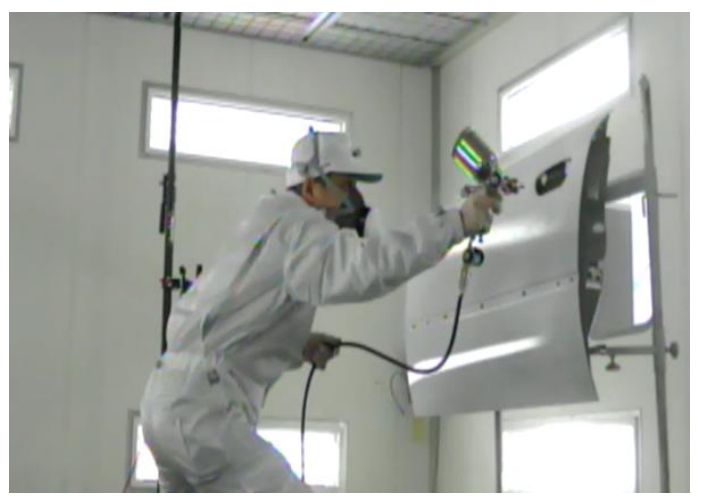

Fig. 1. Photo of block panting work. 

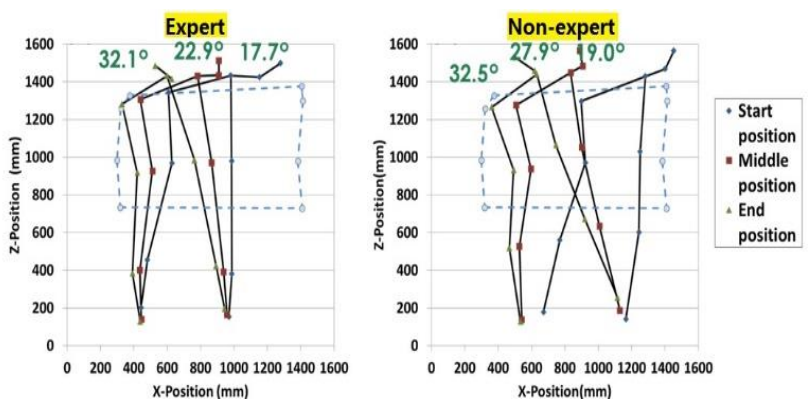

Fig. 2. An example of the posture data [12].
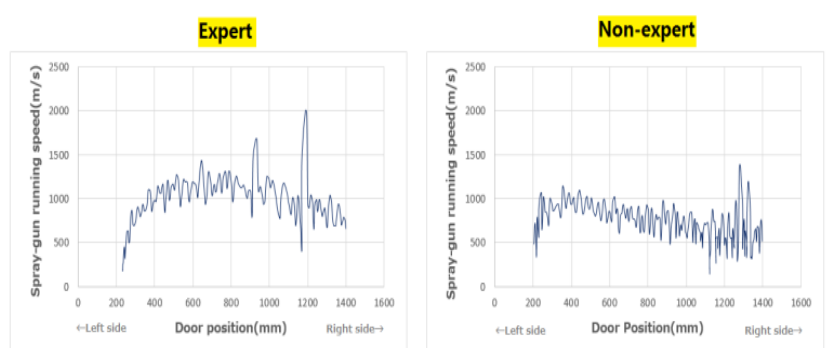

Fig. 3. Relationship between splay-gun running speed and door position [12].

\section{CReAtion of E-LEARning TEACHING MATERIALS}

Before we made e-learning using five items of data determined in 2-3. For animation creation, Smartavatar Creator, moving image creation software developed by BOND limited company, was used. Smartavatar Creator can create animated teaching materials by adding avatar (character to be a part of character) to synthesize speech. The target audience of the e-learning materials is inexperienced engineers and men in their 20s to 30s occupy the majority, so Avatar used girls' anime characters considering their familiarity. The animation was decided to be summarized in about 10 minutes.

Each selected data compares experts and non-experts. The point of work that was particularly important was to display and emphasize telop on the full screen. Fig. 4 shows the screen when telop is displayed. The features of the expert's work taken up in the telop were the following seven points:

1) During work, they stood in the same place without much movement of the feet.

2) They didn't tilt their shoulders too much, but tried to keep it as horizontal as possible.

3) There was a close distance between the expert's waist and the door.

4) The spray gun moved quickly, but slowed down as more tasks got piled.

5) The force to pull the lever that controlled the discharge amount of the spray gun was strong but both left and right edges were loosened.

6) The speed to move the spray gun slowed down at both left and right edges of the door.

7) The film coating was thin and the variation between the maximum and the minimum value of the thickness of the film thickness was small, so the whole panel was finished with a uniform thickness.

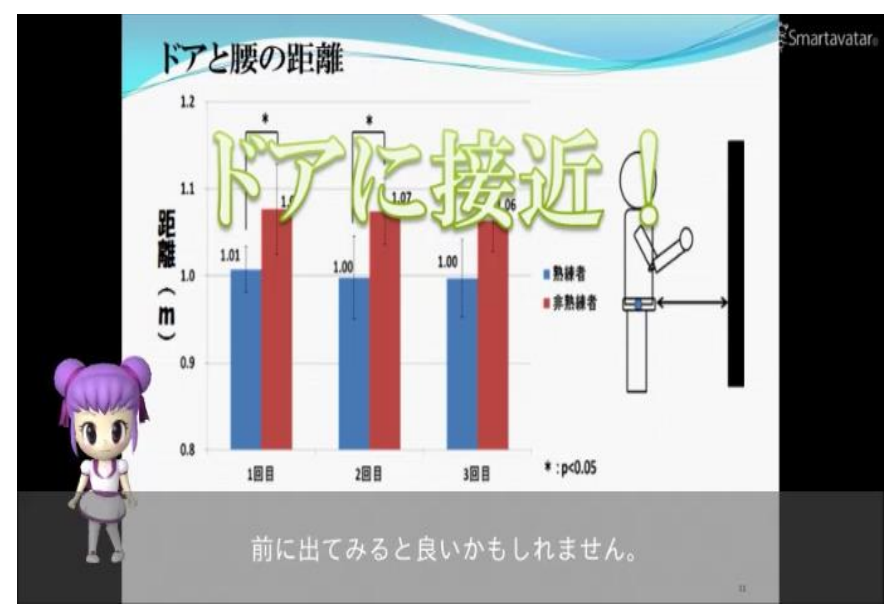

Fig. 4. An example of e-learning materials screen when telop is displayed.

At the end of the e-learning videos, working videos of experts and non-skilled engineers were displayed. The video was paused in the middle, many times, so that the key work movements can be studied and the differences between the work done by experts and non-experts can be explained again so that viewers could reflect.

\section{Publication of E-Learning Materials and AUDIENCE TRENDS}

\section{A. Overview of Video Viewing Evaluation}

The e-learning material was released on Rios Channel [13] which is a video distribution / sharing website specialized in automobile maintenance / sheet metal paint management operated by Protrios Co., Ltd. The Rios channel is a shared site with YouTube where you can learn viewer's viewing situation of the viewer by using Google Analytics and YouTube analytics. The release date of the e-learning material and the analysis period is described below:

Release date: June 12, 2017

Period of analysis: June 12, 2017 - November 11, 2017

Protrios Co., Ltd used facebook and e-mail newsletter for announcement of painting teaching materials. The announcement on facebook took place on June 12. Delivery of the e-mail newsletter was carried out on July 26.

\section{B. Number of Viewers and Audience Trends}

Table I shows the number of users (the actual number of users), the number of page views (the number of views), and the average viewing time in the whole car repair painted elearning teaching material and Rios channels. Based on the number of users, it was found that about $14 \%$ of Rios channel users were watching the e-learning materials. This was the second highest number of accesses to the top page of Rios channel. The number of page views of the e-learning materials was 436 times. Of these, 130 times were by people who viewed it two or more times. The total playing time of the e-learning materials was 8 minutes 57 seconds. In contrast, the average viewing time was 3 minutes 55 seconds. Approximately $72 \%$ of users of e-learning materials were new users. 
TABLE I. The Number of Users, THE Number of PAGe VIEWS, AND THE AVERAGE VIEWING TIME

\begin{tabular}{|l|l|l|l|}
\hline & $\begin{array}{l}\text { Number } \\
\text { of users } \\
\text { (peoples) }\end{array}$ & $\begin{array}{l}\text { Number } \\
\text { of page } \\
\text { views } \\
\text { (times) }\end{array}$ & $\begin{array}{l}\text { Average } \\
\text { viewing time } \\
\text { (h:m:s) }\end{array}$ \\
\hline Painting e-learning material & 316 & 436 & $00: 03: 55$ \\
\hline Rios channels & 2219 & 13228 & $00: 04: 05$ \\
\hline
\end{tabular}

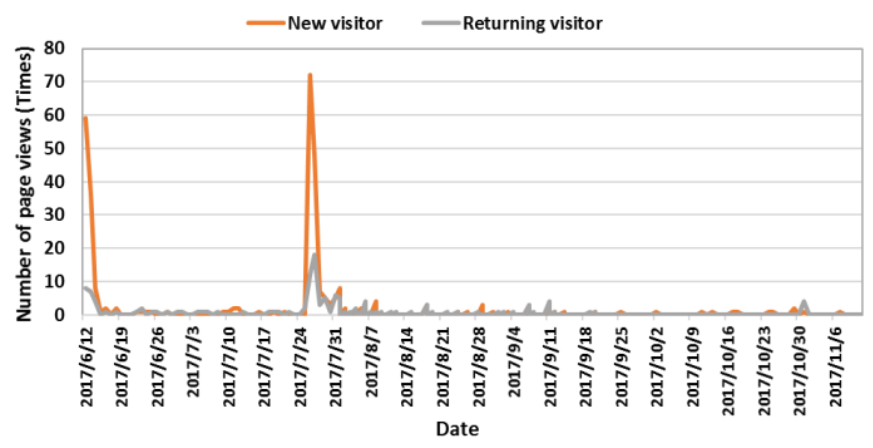

Fig. 5. Relationship between number of page views and date.

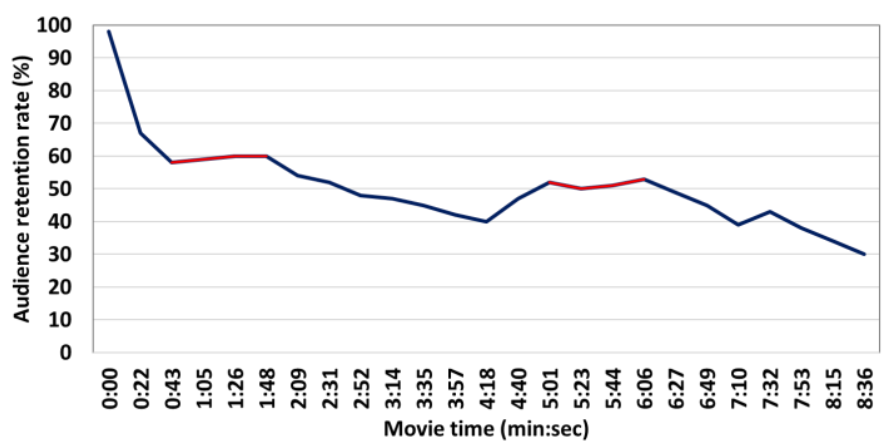

Fig. 6. Audience retention rate.

Fig. 5 shows relationship between number of page views and date. The number of page views showed high values during the movie release date and 2 days thereafter, the e-mail newsletter distribution day and then 6 days. The number of page views on other days was several. The use of new visitors was confirmed even in November. The audience retention rate is shown in Fig. 6. The drop in audience retention rate was small between the first 40 seconds and 1 minute 50 seconds after the start of e-Learning teaching material, and from 5 minutes 00 seconds to 6 minutes 00 seconds. Between the first 40 seconds and 1 minute 50 seconds, there was an explanation regarding the experiment method and posture. And between 5 minutes 00 seconds and 6 minutes 00 seconds, the worker in the video explained the work video about the non-experts.

\section{Viewer's Access Method and Viewing Time Period}

Fig. 7 shows the devices used for viewing the e-learning teaching materials. Desktop computers accounted for $57.6 \%$ of the total. Next, the mobile phone was $39.6 \%$ and the tablet was $2.8 \%$. The viewing time periods of the e-learning material are shown in Fig. 8.

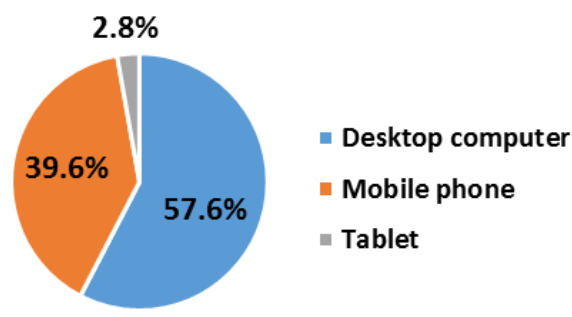

Fig. 7. The devices used for viewing the e-learning teaching materials.

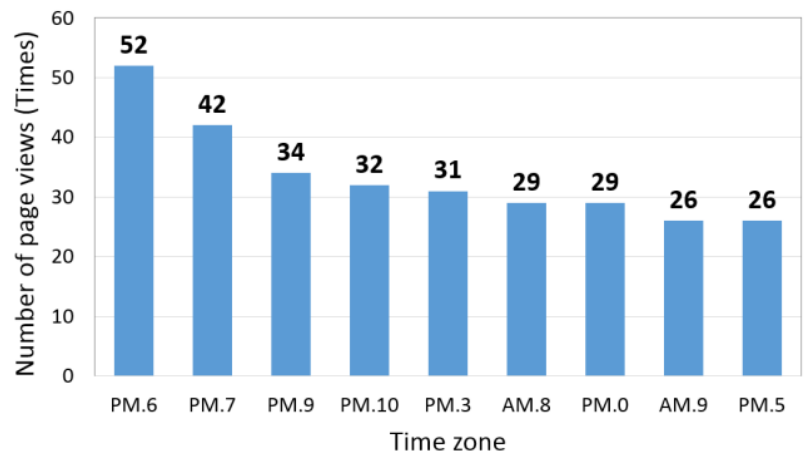

Fig. 8. The viewing time periods of the e-learning material.

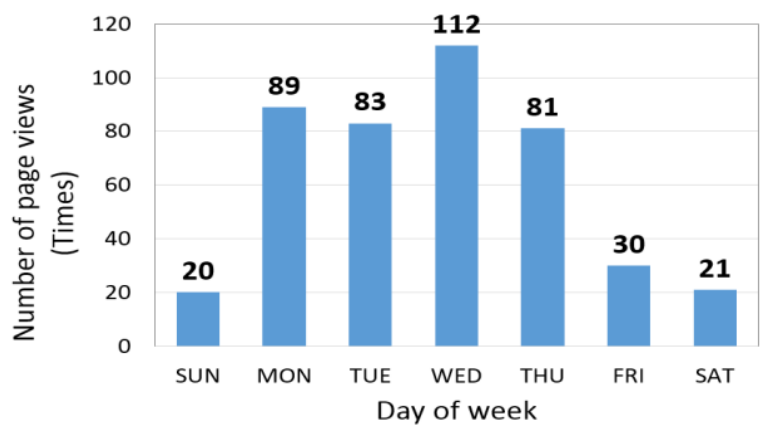

Fig. 9. The viewing days of the e-learning materials.

The most popular time period was at 18 o'clock, accounting for about $12 \%$ of the total. Next to that 19 o'clock and 21 o'clock were also popular viewing time periods. The viewing days of the e-learning materials are shown in Fig. 9. The day of watching which was the most was Wednesdays, accounting for about $26 \%$ of the total. Next, there were many viewers on Mondays and Tuesdays.

\section{DISCUSSION}

The e-learning material produced in this research was 8 minutes 57 seconds, but the average viewing time was 3 minutes 55 seconds. The average viewing time of the whole Rios channel was also 4 minutes 5 seconds, which was not much different from the average viewing time of e-learning material. Therefore, it can be said that the length of a video that an engineer related to car maintenance / repair can most easily watch is about 4 minutes. Moreover, it can be predicted that there are many viewers using e-learning teaching materials in the afternoon of store holidays from the viewing time periods and viewing day of the week. 
Announcement of e-learning material was done only by using Facebook and e-mail newsletter. The fact that we have secured a certain number of returning visitor since the elearning teaching material was released can mean that the elearning has attractive content that viewers wanted to keep watching it.

In the future, by reviewing the relationship between the length and content of e-learning materials and periodically notifying and distributing new teaching materials, it is thought that it may be possible to increase the number of viewers and repeaters. For example, shortening the playback time of an elearning material and dividing the topic to be handled may change the viewing time periods and increase the casual viewing during breaks during work.

From people involved in the automobile repair industry, experiments themselves are grasped favorably; however, there were also opinions that further analysis is required. In this study, we focused only on the block painting work, but we recognize that there are other tasks that rely on experts' skills in the painting work, such as preparation work before pairing, painting colour adjustments on a car and the paint color matching work to match the color of the paint to the actual vehicle. We are also beginning to analyze the other painting methods and the technique of sheet metal work that is often told with painting work, and we are also accumulating new analytical data. Because the support of the automobile repair industry is also great, we would like to improve further teaching materials in the future.

\section{CONCLUSION}

We elucidated the techniques internalized in skilled engineers in block painting work in car repair work, produced e-learning teaching materials for the passing down of skills, and analyzed trends of viewers. The e-learning teaching material secured a certain number of repeaters and was able to use possibly as an effective teaching material. At the same time, several tasks such as shortening teaching material playback time were also highlighted.

\section{ACKNOWLEDGMENT}

We would like to thank the following people for their assistance in conducting this experiment: Mr. Ikemiya of the Kinki branch of the Japan Auto Body Repair Association; Mr.
Ukita of the Nara Prefecture branch of the Japan Auto Body Repair Association; Mr. Tai of the Osaka Prefecture branch of the Japan Auto Body; Mr. Hasegawa, Mr. Oguro, Mr. Aono of Proto-Rios Inc.

\section{REFERENCES}

[1] K. Watanuki, "Virtual training/on-the-job training based skill transfer and human resource development," Journal of the Japan Society for Precision Engineering, vol. 76, Issue 4, pp. 382-389, 2010.

[2] A. Yoshinari, and M. Yamamoto, "Off-the-job-training outside enterprises to meet the needs of small and medium-sized enterprises," Journal of Japan Management Diagnosis Association, vol. 13, pp. 100106, 2013.

[3] Japan Automobile Service Promotion Association., Investigative committee on securing and training personnel for automobile servicing (investigative committee report), 2016, p.1.

[4] Y. Ichimura, "About personnel training of the medium and small-sized business and how to work on workplace communication," Produetion Management, vol. 15, Issue 2, pp. 113-118, 2009.

[5] Y.Kameyama, M. Mizutani, T. Naruse, Y. Koma, C. Sasaki, H. Ohmori, H. Sawada, N. Matsuki, "Development of support tool "process template"to pass on skills and its application for milling of sheet metal," Journal of the Japan Society for Abrasive Technology, vol. 53, Issue 12, pp. 741-744, 2009.

[6] Y. Taguchi, "A study of skill transfer in Japan enterprises," Journal of Shohoku College, (34), pp. 177-187, 2013-03-31.

[7] N. Song, "Methods of the transfer of veteran baby-boomer's skills in the age of information technology," Annals of the Society for Industrial Studies, Japan, vol. 2008, Issue 23, pp. 63-77, 158, 2008.

[8] Y. So, A. Ebe, H. Nakamura, "The development of skill succession support system $(<$ Special Issue $>$ Aiming at knowledge management in production systems)," Systems, Control and Information, vol. 52, Issue 4, pp. 136-141, 2008.

[9] Y. Takai, A. Goto, H. Sato, H. Hamada, "Development of e-learning materials adopting explicit knowledge of expert for Kyoto style earthen wall," Transactions of Japanese Society for Information and Systems in Education, vol. 33, Issue 2, pp. 84-93, 2016.

[10] T. Izumi, Y. Majima, S. Shimada, "Development and evaluation of a new nurse training program which utilizes e-learning," Transactions of Japanese Society for Information and Systems in Education, vol. 31, Issue 1, pp. 57-68, 2014.

[11] Toyota Motor Sales U.S.A Inc., Collision Repair \& Refinish Technicians Course of [http://www.crrtraining.com/CRR2/course-of-study/].

[12] S. Ikemoto, Y. Takai, H. Hamada, "Expert's common factor of painting motion in auto repair painting process," Advances in Ergonomics of Manufacturing: Managing the Enterprise of the Future, vol.490, pp.2333, 2016.

[13] Proto-Rios Inc., RIOS channels, [https://riosch.jp/]. 